\title{
Experimental identification of a thermo-mechanical model for air bending
}

\author{
J.A. Canteli, J.L. Cantero, M.H. Miguélez* \\ Department of Mechanical Engineering, University Carlos III of Madrid, Av. Universidad 30, 28911 Leganés, Madrid, Spain
}

Keywords:

Air bending

High temperature

Sheet metal forming

Modelling

Springback

\begin{abstract}
Experimental validation of thermo-mechanical modelling of air bending at high temperature is presented in this paper. A non-conventional experimental set up is designed and fabricated. Heating device is based on lineal heating along bend length, so temperature distribution is generated in sheet metal, it is previously instrumented to measure temperature. Bending device is based on a commercial press brake instrumented to measure bending force and punch displacement. Bending angle is measured before and after unloading. Model errors, defined by differences between calculated and measured values, were small. Developed work has proved that high temperature bending is easy to implement in industry, and developed model is able to predict accurately process parameters.
\end{abstract}

\section{Introduction}

New requirements in sheet metal forming processes, like high strength materials and accuracy demands, could be achieved forming at elevated temperature and developing accurate models able to predict parameters involved in process (Canteli, 2003). In a previous paper (Canteli et al., 2006) an analytical model for air bending process, at local elevated temperature along bend length, was presented. A brief summary of the model is presented in this paper. A constitutive equation of the material sheet, as a function of temperature was included in the model. Temperature distribution in the sheet was calculated modelling a heating device developed to validate the model.

Several papers dealing with experimental work on air bending processes are found in technical literature. The influence of tool geometry (die aperture and punch radius) and sheet thickness, in process results (specially springback angle), has been analysed for decades (Eary and Reed, 1974; Melin et al., 1982; Jones, 1989; Tan, 1994; Moon et al., 2003). Also mechanical behaviour of the sheet material, is an important factor influencing the process (Inamdar et al., 2002). Other authors have developed analytical and numerical models that had to be experimentally validated (Livatyali and Altan, 2001; Forcellese et al., 1998; Huang and Leu, 1998; de Vin et al., 1996; de Vin, 2000; Asnafi, 2000). These works involved more or less complicated experimental set ups, including measurements systems, sometimes developed to work in process. Although research work about air bending process is extended (de Vin, 2001), no study about this process at high temperature was found. Of course, laser bending process is an exception, but this technique is completely different because strains are the result of the intense local heating, and no punch is involved in the process (Hennige, 2000; Hu et al., 2001).

This paper presents a summary of the thermo-mechanical model for air bending with local heating and the experimen-

\footnotetext{
* Corresponding author. Tel.: +34 9162494 02; fax: +34 916249430.

E-mail address: mhmiguel@ing.uc3m.es (M.H. Miguélez).
} 
tal validation of the analytical model. Thermal model predicts temperature distribution in the sheet, once heating parameters are established. First, a heating device was designed and constructed, based on cheap components and materials. Complex and expensive technologies were avoided in order to obtain a device easy to implement in industry. Mechanical model predicts main parameters in bending process (springback, bending force, punch displacement) once desired final bending angle of the workpiece is defined. Secondly, a conventional NC pressbrake, was modified to carry out bending tests at room and high temperature. All experimental work was done with stainless steel AISI 304, after previous mechanical characterization.

From differences between theoretical and experimental values of main parameters (springback and bending force), errors are calculated showing model accuracy and so, the interest for industrial applications.

\section{Thermo-mechanical model}

\subsection{Temperature distribution}

Temperature distribution was generated in the sheet when it was locally heated along bend length. Thermal model is schematically presented in Fig. 1, only one half of the sheet is represented because of the symmetry with respect to plane $y z$. The sheet was divided in several elements, being each element $\Delta \mathrm{X}$ wide and $1 \mathrm{~m}$ long, and $e$ thick. Uniform temperature $T_{n}$ in each element was assumed. Thermal flows in element $n$ are represented in this figure:

- Conduction flows from element $n-1$ and to element $n+1$ (E $\left.E_{\text {cond } n-1}, E_{\text {cond } n}\right)$.

- Convection flows from element $n$ to air ( $\left.E_{\text {conv }}\right)$.

- Radiation flows from each resistance to element $n$ and from element $n$ to air $\left(E_{\text {radressheet }}, E_{\text {rad }}\right)$.

In element $n=1$ conduction flows with resistances was also considered and resistances convection and radiation flows to air were taken into account.
2.1.1. Temperature in element $n$

With this assumptions expression of temperature in element $n$ in time $t$ was obtained:

$$
\begin{gathered}
\mathrm{T}(\mathrm{t})_{n}=\mathrm{T}(\mathrm{t}-1)_{n}+\frac{\begin{array}{c}
\Delta E_{\text {cond }}-\Delta E_{\text {conv }}-\Delta E_{\text {rad }} \\
+\Delta E_{\text {radres-sheet1 }}+\Delta E_{\text {radres-sheet2 }}
\end{array}}{C\left(T(t-1)_{n}\right) \rho_{\text {sheet }} \Delta x e} \Delta t
\end{gathered}
$$

where $\rho_{\text {sheet }}$ is the material density, $C(T)$ the specific heat (dependent on temperature), $\Delta E_{\text {cond }}$ the energy increment (in each time unit) due to conduction flows, $\Delta E_{\text {conv }}$ the energy decrement (in each time unit) in element $n$ due to convection flow to air, $\Delta E_{\text {rad }}$ the energy decrement (in each time unit) in element $n$ due to radiation from element to air and $\Delta E_{\text {rad-sheet } 1,2}$ is the energy increment (in each time unit) in element $n$ due to radiation from resistances. Detailed equations for these thermal flows can be found in Canteli (2003).

\subsubsection{Resistances temperature}

Initial temperature in resistances $T_{\text {res }}$ (before contact with sheet) was calculated as a function of heating parameters. Total power in resistances is dissipated in radiation and convection. Relation between radiation power $\left(\mathrm{W}_{\text {rad }}\right)$ and convection power $\left(\mathrm{W}_{\text {conv }}\right)$ and temperature in resistances is

$$
\begin{aligned}
\mathrm{W}= & \mathrm{W}_{\text {rad }}+\mathrm{W}_{\text {conv }}=2 \pi R\left[\sigma \varepsilon_{\text {res }}\left(\mathrm{T}_{\text {res }}^{4}-\mathrm{T}_{\text {room }}^{4}\right)\right. \\
& \left.-\bar{h}\left(\mathrm{~T}_{\text {res }}-\mathrm{T}_{\text {room }}\right)\right]
\end{aligned}
$$

Equation was solved with iterative Newton method.

Contact thermal resistance between resistances and sheet was established from experimental work. Energy increment in first element was

$$
\Delta E_{\text {Cond }}=\frac{1}{R} \frac{\delta}{2}\left(T_{\text {res }}-T_{0}\right)
$$

where $\delta$ contact area and $\mathrm{T}_{0}$ initial temperature in first element.

Resistances temperature in time $t$ was calculated as

$$
\begin{aligned}
T(t)_{\text {res }}=T(t-1)_{\text {res }}+ & \frac{-\Delta E_{\text {cond }}-\Delta E_{\text {rad-sheet }}-\Delta E_{\text {rad-air }}}{C\left(T(t-1)_{\text {res }}\right) \rho_{\text {res }} \pi\left(R^{2} / 2\right)}
\end{aligned}
$$

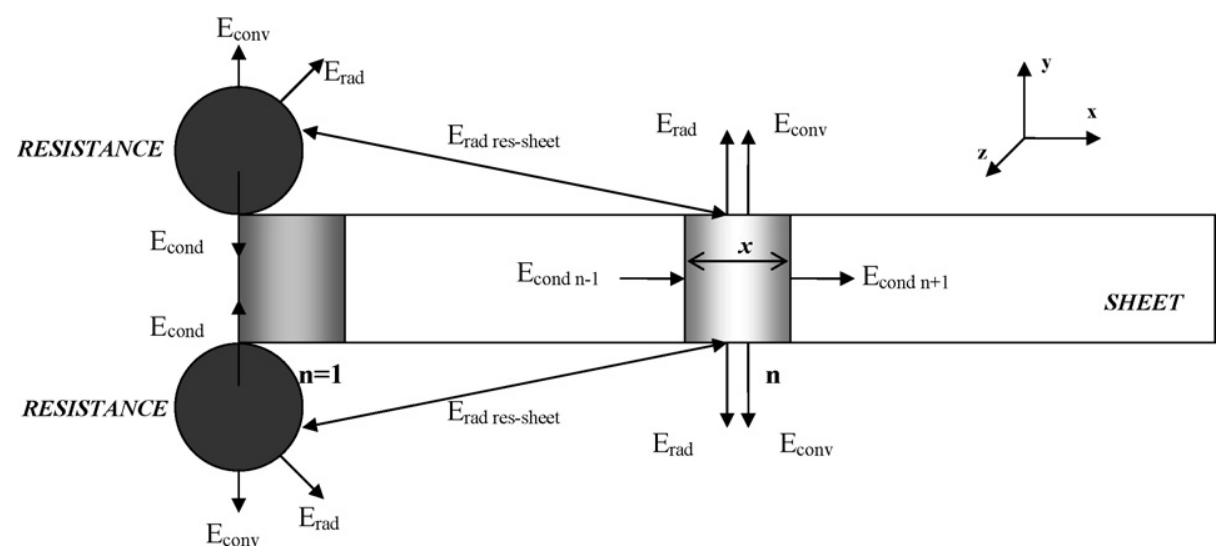

Fig. 1 - Scheme of thermal flows in each element. 
where $\Delta E_{\text {rad-sheet }} \Delta E_{\text {rad-air }}$ and $\Delta E_{\text {conv }}$ energy decrement in resistances because of radiation to sheet and air and convection to air, respectively. Detailed equations for these thermal flows can be found in Canteli (2003).

Eqs. (1)-(4) were used to calculate temperature distribution in sheet and resistances in each time. Time increment was selected according to stability requirements being:

$\Delta t \leq \frac{\Delta x^{2}}{2 \alpha}=\frac{(\Delta x)^{2} \rho c_{p}}{2 k}$

The choice of $\Delta x$ was based on a compromise between accuracy and computational requirements.

Once temperature distribution was obtained, it was possible to estimate mechanical properties in each element, since parameters of constitutive equation are a function of temperature.

\subsection{Bending process at high temperature}

Air bending model symmetrical with respect to bending plane (YZ), is schematically represented in Fig. 2. Central section of the sheet was attached and the contact force die-sheet is applied in contact point $\left(X_{k}, Y_{k}\right)$.

The sheet was divided in elements, in the same way as it was done in thermal model. Some hypotheses were formulated to simplify the problem. During punch displacement, reaction force between die and sheet $F$, varies, in module and direction, as the sheet is being bent. Point of application of this force in the sheet is also variable and so on the effective die aperture. Friction between sheet and die is taken into account.

Moment in a generic element $n$ with central point coordinates $\left(X_{n}, Y_{n}\right)$ is given by

$M_{n}=F\left\{(\sin \varphi-\mu \cos \varphi)\left(Y_{k}-Y_{n}\right)+(\cos \varphi+\mu \sin \varphi)\left(X_{k}-X_{n}\right)\right\}$

where $\mu$ is the friction coefficient between sheet and die $\left(X_{k}\right.$, $Y_{k}$ ) are the co-ordinates of sheet-die contact point, $F$ the normal force due to this contact and $\varphi$ is the angle related to bending angle $\alpha$ as $\varphi+(\alpha / 2)=\pi / 2$.

This external moment should be in equilibrium with internal moments. Internal moment for bending without tension was calculated with several assumptions. In thin sheets, normal section planes may be considered to remain plane on

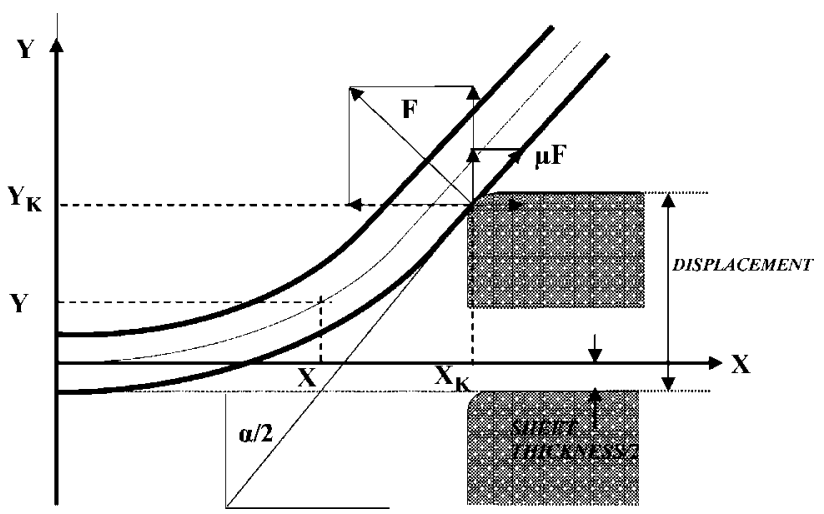

Fig. 2 - Scheme of mechanical model for air bending.

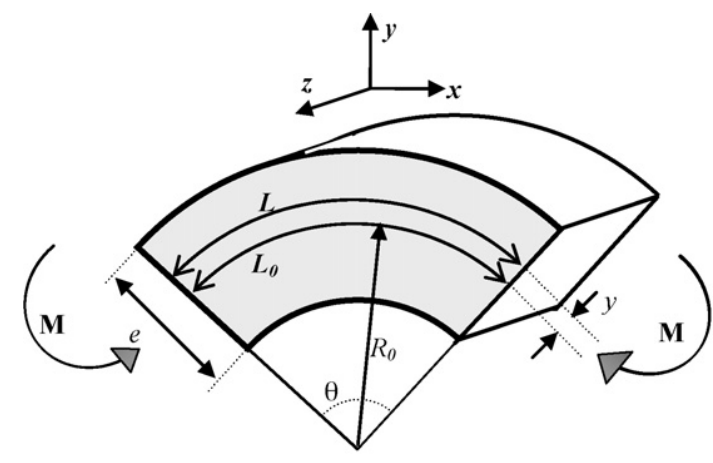

Fig. 3 - Element $n$ during bending.

bending and to converge on the centre of curvature as illustrated in Fig. 3, representing a generic element $n$. Constant curvature was assumed in each element. It was also considered that there is no shear in the radial plane and gradients of stress and strain are zero in the circumferential direction and plane strain conditions were assumed. With these assumptions engineering strain was calculated as

$e_{x}=\frac{L-L_{0}}{L_{0}}=\frac{y \theta}{R_{0} \theta}=\frac{y}{R_{0}}$

Subscript 0 denotes magnitudes in middle surface $\left(R_{0}\right.$ is radius in middle surface) and $x, y, z$ are local axis in element $n$. True strain $\varepsilon_{X}$ in direction $x$ is

$\varepsilon_{x}=\ln \left(1+\frac{y}{R_{0}}\right) \cong \frac{y}{R_{0}}$

From Levy-Mises (Khan and Huang, 1995) equations (increasing monotonic load is considered) effective strain and stress $\left(\varepsilon_{\mathrm{e}}, \sigma_{\mathrm{e}}\right)$ are calculated:

$\varepsilon_{\mathrm{e}}=\frac{2 \varepsilon_{x}}{\sqrt{3}}$

$\sigma_{\mathrm{e}}=\frac{\sigma_{x} \sqrt{3}}{2}$

Moment equilibrium in element $n$ implies:

$M_{n}=\int_{-e / 2}^{+e / 2} \sigma_{x} y d y$

In this equation $\sigma_{\mathrm{x}}$ is related with $\sigma_{\mathrm{e}}$ and so with $\varepsilon_{\mathrm{e}}$, by material constitutive equation.

In elastic region, constitutive equation is

$\sigma_{\mathrm{e}}=\mathrm{E} \varepsilon_{\mathrm{e}}$

And in elastoplastic region:

$\sigma_{\mathrm{e}}=\sigma_{0 \mathrm{e}}+K_{\mathrm{e}} \varepsilon_{\mathrm{e}}$ 
Taking into account these considerations and equations, expression for $\sigma_{x}$ in elastic region, could be written as

$\sigma_{x}=\frac{E \varepsilon_{X}}{1-v^{2}}=\frac{E y}{\left(1-v^{2}\right) R_{0}}$

where $v$ is Poisson coefficient.

In elastoplastic region equation for $\sigma_{X}$ is

$\frac{\sqrt{3}}{2} \sigma_{x}=\sigma_{0 \mathrm{e}}+\frac{2 \mathrm{~K}_{\mathrm{e}} \varepsilon_{\mathrm{x}}}{\sqrt{3}}$

And so

$\sigma_{\mathrm{x}}=\frac{2 \sigma_{0 \mathrm{e}}}{\sqrt{3}}+\frac{4 K_{\mathrm{e}} \varepsilon_{\mathrm{x}}}{3}=\frac{2 \sigma_{0 \mathrm{e}}}{\sqrt{3}}+\frac{4 K_{\mathrm{e}} \mathrm{y}}{3 \mathrm{R}_{0}}$

From Eqs. (11), (14) and (16) the bending moment can be expressed as follows:

$M_{n}=2\left[\int_{0}^{\varepsilon_{x}^{*} R_{0}} \frac{E}{1-v^{2}} \frac{y^{2}}{R_{0 n}} d y+\int_{\varepsilon_{x}^{*} R_{0}}^{e / 2} \frac{2}{\sqrt{3}} \sigma_{0 e}+\frac{4 K_{e}}{3} \frac{y}{R_{0 n}} y d y\right]$

Where integration limits in equation are given by the point where yielding begins, corresponding to strain $\varepsilon_{x}^{*}$ given in Eq. (18):

$\varepsilon_{\mathrm{x}}^{*}=\frac{\sigma_{0 \mathrm{e}}\left(1-v^{2}\right)}{\left(E-K_{\mathrm{e}}\right) \sqrt{1-v+v^{2}}}$

Integration of Eq. (17) relates $M_{n}$ with curvature $R_{0 n}$ in element $n$ :

$$
\begin{aligned}
M_{n}= & \left(R_{0 n} \varepsilon_{x}^{*}\right)^{2}\left[\frac{2}{3} \varepsilon_{x}^{*}\left(\frac{E}{1-v^{2}}-\frac{4}{3} K_{e}\right)-\frac{2}{\sqrt{3}} \sigma_{0 e}\right] \\
& +0.2887 \sigma_{0 e} e^{2}+\frac{K_{e} e^{3}}{9 R_{0 n}}
\end{aligned}
$$

As constitutive equation parameters $\left(E(T), \sigma_{0 e}(T), K_{e}(T)\right)$ depends on temperature T, Eq. (19) should be formulated once temperature in each element is calculated, as was explained previously.

Moment calculated from Eq. (19) should be equal to moment deduced from external forces given in Eq. (6).

Eq. (20) relating curvature in each element and $Y(X)$ is given by Timoshenko (2002):

$$
\frac{1}{R_{0 n}}=\frac{\left(d^{2} Y / d X^{2}\right)_{n}}{\left[1+(d Y / d X)_{n}^{2}\right]^{3 / 2}}
$$

Final curvature after unloading $1 / R_{\mathrm{fn}}$ is calculated as

$$
\frac{1}{R_{0 n}}-\frac{1}{R_{\mathrm{fn}}}=\frac{M_{n}}{E e^{3} / 12\left(1-v^{2}\right)}
$$

And final $Y(X)$ of the sheet (in each element $n$ ) after unload, is related to final curvature as follows:

$$
\frac{1}{R_{f n}}=\frac{\left(d^{2} Y / d X^{2}\right)_{n}}{\left[1+(d Y / d X)_{n}^{2}\right]^{3 / 2}}
$$

In a first step $R_{0}^{*}$, radius where yielding occurs in $y= \pm(e / 2)$, was calculated as follows:

$\mathrm{R}_{0}^{*}=\frac{e \sqrt{3}}{4 \sigma_{0 \mathrm{e}}}\left(\frac{E}{1-v^{2}}-\frac{4}{3} K_{\mathrm{e}}\right)$

As it has been shown, moment resulting from external forces was calculated (Eq. (6)). Parameters $F, \varphi, X_{k}, Y_{k}$ were not known, and were arbitrarily fixed, as a first step of the iterative process.

This moment in element $n$, should be equal to internal moment resulting from Eq. (19). Newton method (Bilal and McCuen, 1996) was used to calculate $R_{0 n}$ from this equation. If $R_{0 n}<R_{0}^{*}$ (elastoplastic) then $R_{0 n+1}$ was calculated in a similar way. If $R_{0 n} \geq R_{0}^{*}$ (elastic) then $R_{0 n}$ was recalculated as

$R_{0 n}=\frac{E}{1-v^{2}} \frac{1}{M_{n}} \frac{e^{3}}{12}$

From Eq. (21) $Y(X)$ and its derivatives were obtained. Boundary conditions were $\mathrm{Y}=0$ and $(\mathrm{d} Y / \mathrm{dX})=0$ in $X=0$, and solving method is fourth order Runge-Kutta (Hultquist, 1988).

Then, bending angle before springback, was calculated. When unloading occurs, final curvature in each point after springback was calculated as

$\frac{1}{R_{\mathrm{f} n}}=\frac{1}{R_{0 n}}-\frac{12\left(1-v^{2}\right) M_{n}}{E e^{3}}$

Relation between curvature and final $Y(X)$ is given by Eq. (22). This equation was also solved by a fourth order Runge-Kutta method with contour conditions $Y=0$ and $(d Y / d X)=0$ in $X=0$. Resultant bending angle after springback was calculated. Differences between estimated and calculated values indicate if it was necessary to iterate again. The procedure was stopped once a convergence criterion was reached.

\section{Experimental set up}

In this paragraph, equipments designed and constructed to validate thermo-mechanical model are described.

\subsection{Heating device}

Heating device was designed and constructed to heat the sheet in a narrow area along bend length. It is clear that local heating presents advantages, when it is compared to complete introduction of the sheet in a furnace at high temperature: easier handling of hot sheet, smaller and less expensive equipments, local instead extended oxidation and energy saving, mainly.

Heating device was based on two standard electrical resistances (cylindrical shape, radius $6.3 \mathrm{~mm}$, length $400 \mathrm{~mm}$, power range from 900 to $2000 \mathrm{~W} / \mathrm{m}$ ), mounted in a test set as is shown in a scheme in Fig. 4. Once resistances were heated up to a desired high temperature, sheet was located between both resistances and contact force was applied (contact force ranged from 375 to $750 \mathrm{~N} / \mathrm{m}$ ). The axis of resistances and the line that was going to be bent (after heating) were in the same plane.

After heating time long enough to ensure permanent conditions in the sheet ( $20 \mathrm{~min}$, in the case of thickest sheets), it 


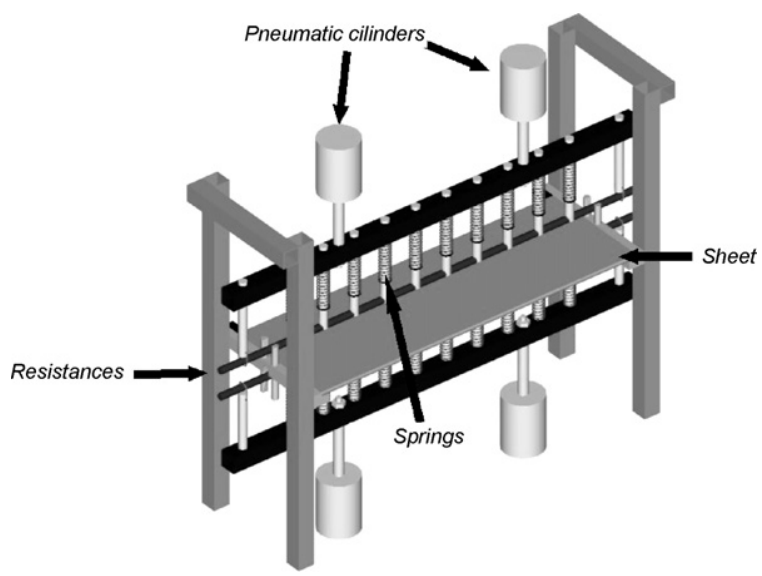

Fig. 4 - Scheme of heating device.

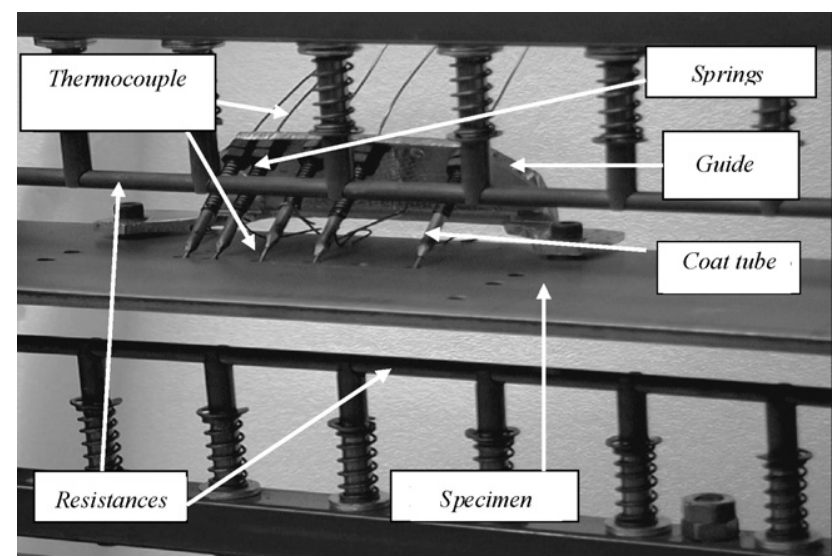

Fig. 5 - Thermocouples installed to validate temperature distribution in the sheet.

should be positioned in the pressbrake to carry out bending tests. Experimental set up was designed to allow quickly positioning of the sheet in the pressbrake avoiding temperature decrease.

As temperature should be measured during thermal tests, sheet was instrumented with thermocouples as shown in Fig. 5. Selected sheet thickness for thermal model validation was $6 \mathrm{~mm}$ because installation of thermocouples required thick sheet.

Thermal test indicated temperature distribution in the sheet, once thermal parameters (resistances power before and after contact, heating time and contact force between resistance and sheet) were fixed. Maximum temperatures up to $770^{\circ} \mathrm{C}$ along bend length (corresponding to maximum contact forces and power resistances) were obtained. Comparison between measured and calculated temperatures allowed obtaining errors in analytical predictions.

As it was not possible to bend sheet instrumented with thermocouples, bending tests were carried out with not instrumented sheets heated with exactly the same parameters. Fig. 6 is a photograph of equipment during heating phase of a sheet without thermocouples, previously to bending phase.

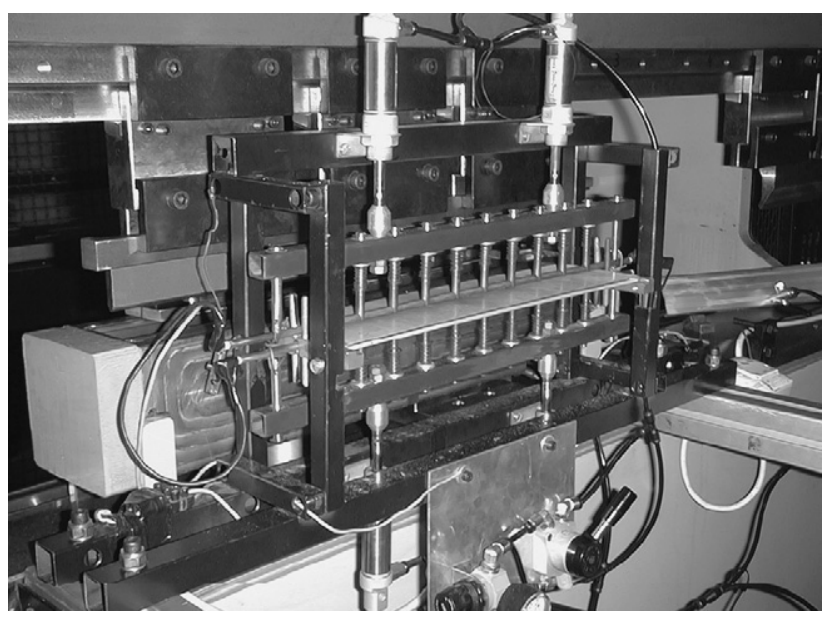

Fig. 6 - Sheet positioned on heating device before bending.

\subsection{Bending device}

Machine for bending tests was a conventional hydraulic pressbrake LOIRE-SAFE with numerical control CYBELEC, being its maximum bend length $3050 \mathrm{~mm}$ and maximum nominal force $900 \mathrm{kN}$

This machine control punch displacement and speed during bend tests. Punch position was also measured with a displacement transducer and compared with internal displacement control of the NC machine. Signal from displacement transducer was distorted because of noise, however, medium value of displacement signal, coincide reasonably with signal obtained from machine displacement controller. Punch displacement was obtained from internal control of the machine.

Standard tools were substituted by specially designed and constructed tools that allowed ranging geometric parameters (punch radius, die aperture) and sheet geometry in desired intervals. Sheet thickness ranged from 2 to $6 \mathrm{~mm}$, punch radius ranged from 1 to $4 \mathrm{~mm}$ and die aperture from 6 to $12 \mathrm{~mm}$ times the sheet thickness. Bend length of the sheet was enough to ensure plane strain conditions in the sheet.

A load cell was installed as a support of constructed dies. As the process is symmetrical one half of bending force is measured as is shown in Fig. 7. Hydraulic system of pressbrake was instrumented with a pressure sensor, obtaining another measure of bending force. Results obtained from both systems were coincident.

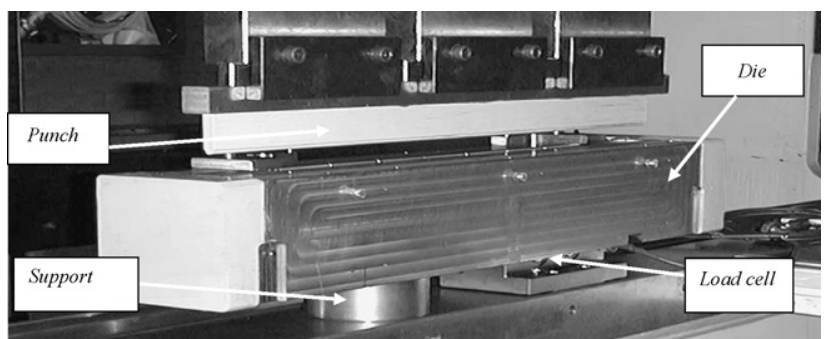

Fig. 7 - Load cell installed in modified die to measure bending force. 


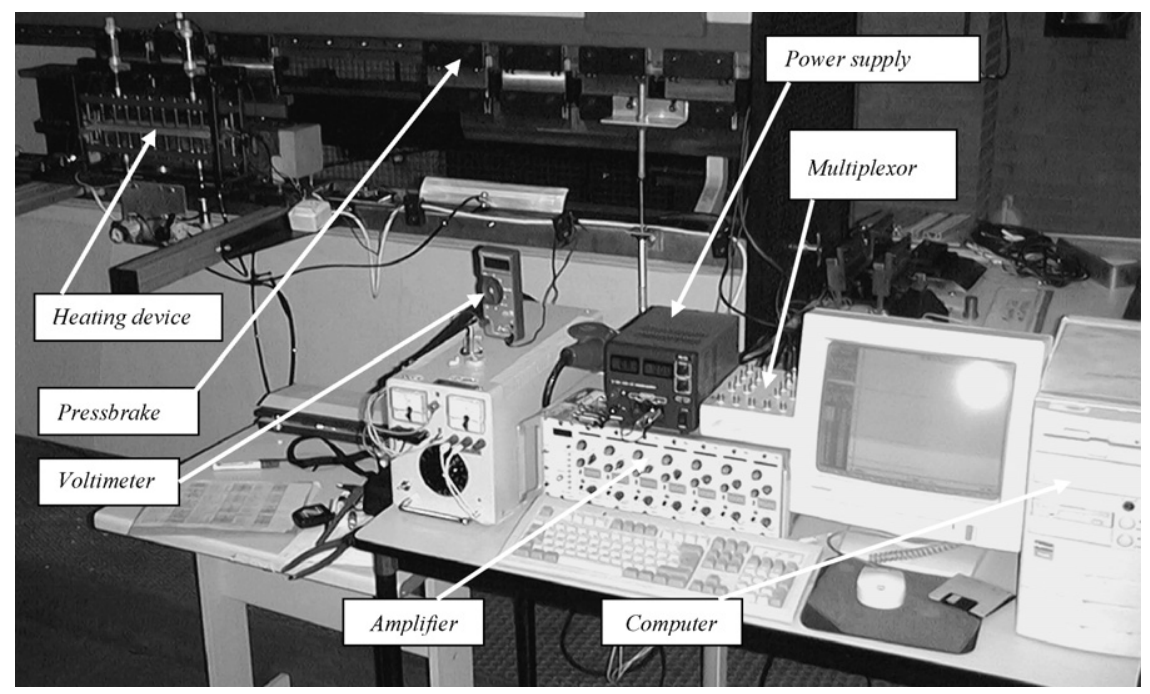

Fig. 8 - Complete experimental set up with acquisition system.

Bending angle was measured in the maximum displacement of the punch and after unloading, with a goniometer.

Pressbrake and complete acquisition system is shown in Fig. 8.

\section{Experimental results}

In this paragraph experimental work developed to validate thermo-mechanical model is described. First tests for validation of theoretical temperature distribution are summarized, and secondly bending tests at both room and elevated temperature are presented. Differences between theoretical and experimental values indicate the accuracy of the model.

\subsection{Validation of thermal model}

Thermal model calculates temperature in a generic element $n$ (located at a distance $x$ from bending line), as was explained previously.

From laboratory tests, temperatures in three points were obtained. Thermocouples were installed in sheet points with coordinate $x=0,2$ and $4 \mathrm{~mm}$. Model validation was based on the comparison of calculated and measured temperatures in these three points at four different values of time $5,10,15$ and $20 \mathrm{~min}$. Measured temperatures in coordinates $x=0,2$ and $4 \mathrm{~mm}$ were named $\mathrm{T}_{0}, \mathrm{~T}_{2}$ and $\mathrm{T}_{4}$. Calculated temperatures in coordinates $x=1,3$ and $5 \mathrm{~mm}$, corresponding to middle point of elements $n=1,2$ and 3 , were named $T_{1}, T_{3}$ and $T_{5}$. Selected $\Delta x$ was $2 \mathrm{~mm}$, as a compromise between accuracy and time of calculus. Lineal interpolation and extrapolation are used to compare theoretical and experimental temperatures in coordinates $x=1$ and 3 and in coordinate $x=5$, respectively (see Eq. (1)). Mean error is calculated in these three points in each value of time $(5,10,15$ and $20 \mathrm{~min})$ and mean of these four errors is calculated as shown in Eq. (26):

$$
\begin{aligned}
& \begin{aligned}
t & =20 \\
e=\frac{1}{4} \sum_{t}^{t}=15 & \frac{1}{3}\left[\frac{\left|\left(\left(T_{0}+T_{2}\right) / 2\right)-T_{1}\right|}{\left(T_{0}+T_{2}\right) / 2}+\frac{\left|\left(\left(T_{2}+T_{4}\right) / 2\right)-T_{3}\right|}{\left(T_{2}+T_{4}\right) / 2}\right. \\
t & =5
\end{aligned} \\
& \left.+\frac{||\left(\left(3 \mathrm{~T}_{4}-\mathrm{T}_{2}\right) / 2\right)\left|-\mathrm{T}_{5}\right|}{\left|\left(3 \mathrm{~T}_{4}-\mathrm{T}_{2}\right) / 2\right|}\right]
\end{aligned}
$$

As an example, error is calculated in the case of maximum contact force and maximum power in resistances, equivalent to maximum temperature distribution in the sheet. Measured and calculated temperatures are summarized in Table 1. Calculated medium error (based on Eq. (1)) was inferior to $0.43 \%$.

In the same way errors were calculated for different combinations of contact force and resistances power, with the

Table 1 - Summary of measured and calculated temperatures (in four time values), being heating parameters maximum

\begin{tabular}{|c|c|c|c|c|c|c|}
\hline \multirow[t]{2}{*}{ Time (min) } & \multicolumn{6}{|c|}{ Temperatures $\left({ }^{\circ} \mathrm{C}\right)$} \\
\hline & Measured $(x=0)$ & Calculated $(x=1)$ & Measured $(x=2)$ & Calculated $(x=3)$ & Measured $(x=4)$ & Calculated $(x=5)$ \\
\hline 5 & 640 & 633 & 624 & 617 & 608 & 601 \\
\hline 10 & 735 & 728 & 720 & 709 & 698 & 691 \\
\hline 15 & 774 & 764 & 759 & 744 & 731 & 726 \\
\hline 20 & 790 & 785 & 774 & 765 & 747 & 745 \\
\hline
\end{tabular}
contact force and maximum power resistance 
same width element $(2 \mathrm{~mm}$ ) that gives reasonably calculation time. Maximum temperature in the sheet ranged from $580^{\circ} \mathrm{C}$ (corresponding to low power and low contact force) to $770^{\circ} \mathrm{C}$ (corresponding to high power and high contact force).

Estimated errors were always less than $2 \%$.

\subsection{Validation of bending model}

Mechanical model predicts punch displacement and bending force once bending angle is fixed. Other interesting parameters like stress and strain distribution can be obtained from the model.

In validation tests, calculated punch displacement was imposed in the pressbrake and bending force, and final angle before and after unloading were measured. Validation is based on comparison between measured and calculated values.

Model was adjusted by changes in friction coefficient. Variation in this parameter, results in changes in calculated punch displacement and bending force. The friction coefficient was selected when measured and calculated maximum force coincided in the same punch displacement. Error was obtained comparing calculated force as a function of punch displacement with measured force. Medium error was estimated as media of relative errors in points corresponding with 20, 40, 60 and $80 \%$ of total punch displacement, an interval around displacement related to maximum force.

On the other hand, calculated and measured springback angles calculated as relative variation in bending angle after $\left(\alpha_{\mathrm{f}}\right)$ and before $\left(\alpha_{0}\right)$ unloading (Eq. (27)) were compared too and errors were calculated as relative difference between theoretical and experimental values:

$\operatorname{springback}(\%)=\frac{\alpha_{\mathrm{f}}-\alpha_{0}}{\alpha_{0}} \times 100$

Two steps were considered in the process: validation of bending model at room temperature and validation of bending model with temperature distribution in the sheet.

\subsubsection{First step: validation at room temperature}

In this phase, model predictions at room temperature were checked. As an example Fig. 9 shows measured and calculated bending force versus punch displacement in the case of thickness $6 \mathrm{~mm}$ and die aperture $48 \mathrm{~mm}$. Good agreement between calculated and measured values is observed. The mean error (estimated as the mean of relative errors in four points) was lower than $2 \%$.

Tests covered ranges of sheet thickness, punch radius and die aperture, described in previous paragraph. Calculated errors in all cases were always less than $5 \%$.

The influence of tools geometry was also analysed. Bending force was calculated and measured with different sheet thickness when punch radius ranged from 1 to $4 \mathrm{~mm}$. As an example the same case (thickness $6 \mathrm{~mm}$ and die aperture $48 \mathrm{~mm}$ ) is presented with three different punch radius: 2, 3 and $4 \mathrm{~mm}$. In Fig. 10, it is observed that punch radius influence could be ignorated, in ranges analysed. Calculated errors varied in this case from 1.5 to $3 \%$. Similar values were obtained in another cases checked.

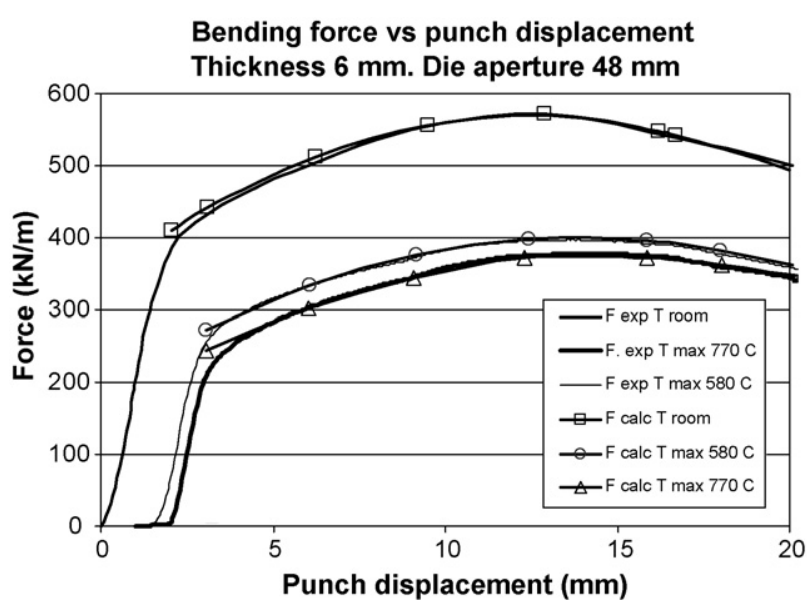

Fig. 9 - Calculated and experimental bending force vs. punch displacement at 770 and $580^{\circ} \mathrm{C}$ and room temperature.

However it is clear that die aperture has great influence in bending force. Each case of sheet thickness (2, 3, 4 and $6 \mathrm{~mm})$ was studied at different die apertures $(6,8,10$ and $12 \mathrm{~mm}$ thickness). Fig. 11 shows an example obtained with sheet thickness $3 \mathrm{~mm}$, and apertures 24, 30 and $36 \mathrm{~mm}$. A relative decrement of $40 \%$ is obtained when aperture is incremented from 8 to 12 times the sheet thickness. In this case medium error ranged from 1.5 to $4 \%$. Similar values were obtained in another cases checked.

As a resume, in bending force calculation at room temperature, maximum errors are always lower than $5 \%$.

In each test described bending angle before $\left(\alpha_{0 \mathrm{~m}}\right)$ and after unloading $\left(\alpha_{\mathrm{fm}}\right)$ were measured. Corresponding model analysis resulted in calculated angle before $\left(\alpha_{0 c}\right)$ and after unloading $\left(\alpha_{\mathrm{fc}}\right)$. Experimental and analytical springback (Eq. (2)) were obtained from bending angle before and after unloading in each test. Error was calculated as relative difference

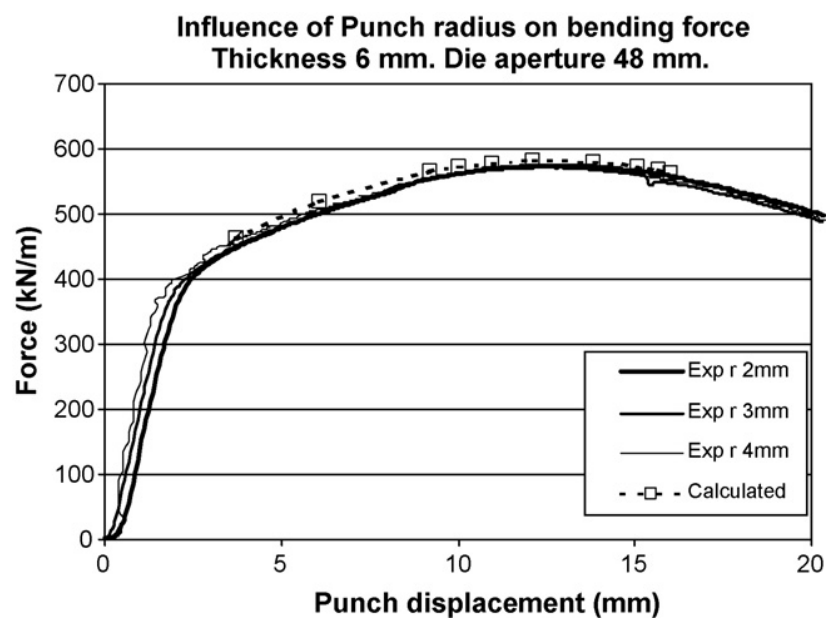

Fig. 10 - Influence of punch radius $(2,3$ and $4 \mathrm{~mm})$ on calculated and experimental bending force obtained at room temperature. 


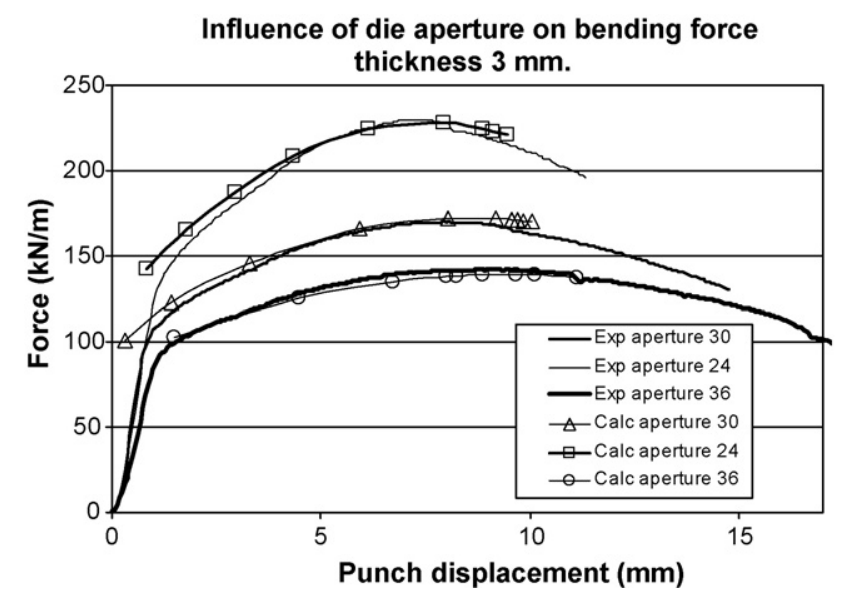

Fig. 11 - Influence of die aperture $(24,30$ and $36 \mathrm{~mm})$ on calculated and experimental bending force obtained at room temperature.

between analytical and experimental values. Several tests, with different bending angle, are summarized in Fig. 12 showing calculated and measured springback versus final bending angle before unloading, when thickness was $4 \mathrm{~mm}$ and die aperture $32 \mathrm{~mm}$. In this case calculated error was less than $0.25 \%$. Similar graphs were obtained ranging sheet thickness.

Summarizing, error in springback angle was inferior to $0.65 \%$.

\subsubsection{Second step: validation at high temperature}

In this second phase, model predictions at high temperature are evaluated. The process is similar to validation at room temperature but in this phase sheets with a known temperature distribution were bent.

Fig. 9 shows bending force versus punch displacement (sheet $6 \mathrm{~mm}$ thick, die aperture $48 \mathrm{~mm}$ ) at room temperature, with temperature distribution corresponding to a maximum

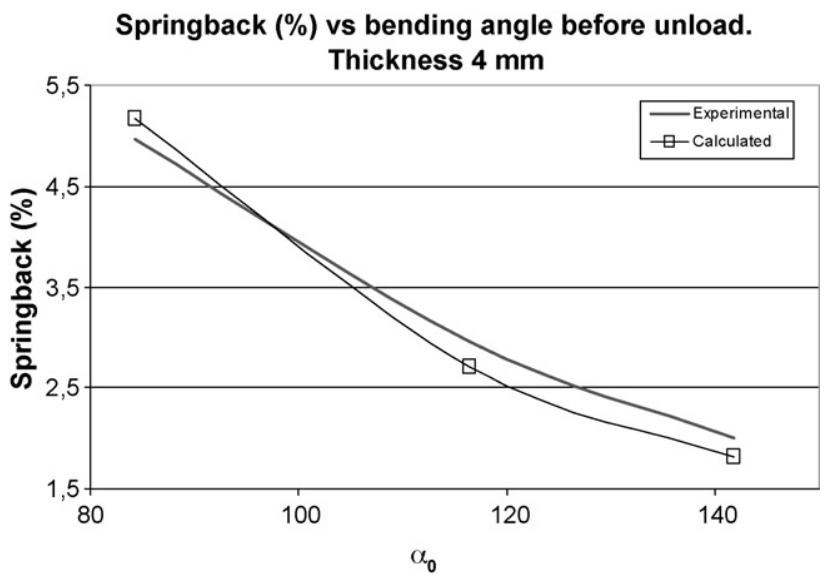

Fig. 12 - Experimental and calculated springback (\%) vs. bending angle before unload at room temperature (die aperture $32 \mathrm{~mm}$ ).

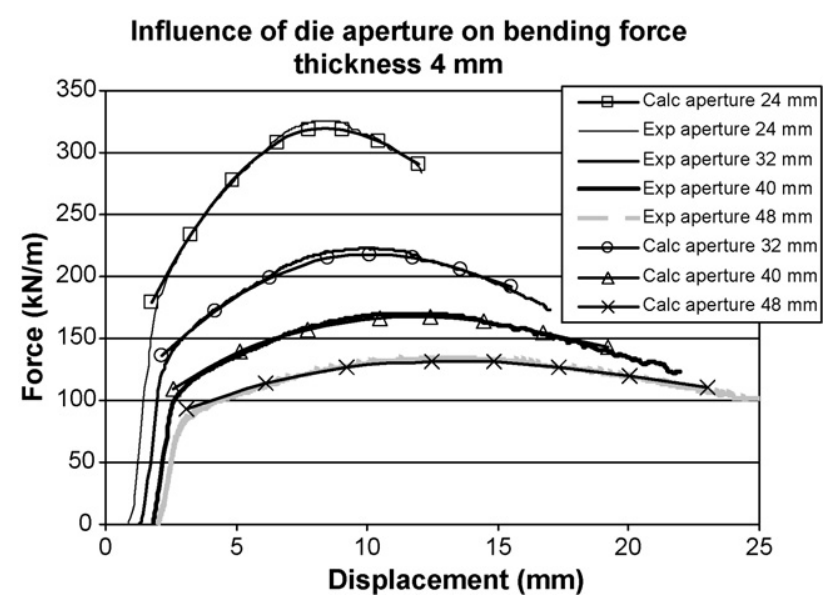

Fig. 13 - Influence of die aperture $(24,32,40$ and $48 \mathrm{~mm})$ on calculated and experimental bending force obtained at maximum temperature $770^{\circ} \mathrm{C}$.

of $580^{\circ} \mathrm{C}$ (obtained with low power resistances and low contact force) and with temperature distribution corresponding to a maximum of $770^{\circ} \mathrm{C}$ (obtained with high power resistances and high contact force). Pronounced influence of temperature in bending force was observed. Maximum bending force decreases as temperature increases, up to decrements of $33 \%$, associated to maximum temperature $\left(770^{\circ} \mathrm{C}\right.$ along bend length).

Error was calculated as was explained previously, obtaining a medium error inferior to $1.5 \%$ in both temperature distributions. Rest of parameters varied in described intervals and tests were carried out at temperatures ranging from 580 to $770^{\circ} \mathrm{C}$. Errors were always less than $5 \%$.

Although it was shown in validation at room temperature that punch radius does not influence bending force, several tests were carried out with different punch radius. The results were in agreement with previous conclusion, and no variation in bending force at high temperature was obtained when punch radius varied.

The effect of die aperture was also evaluated at high temperature. As an example, Fig. 13 shows measured and calculated force with thickness $4 \mathrm{~mm}$ and apertures $6,8,10,12$ times sheet thickness. These curves correspond to maximum temperature distribution (up to $770^{\circ} \mathrm{C}$ ). A great decrement in bending force with die aperture increment is observed. Error was less than $2 \%$.

The influence of die aperture was analysed in the rest of the cases, obtaining similar results.

As a resume, in bending force calculation at high temperature, maximum errors were less than $5 \%$.

Changes in mechanical properties due to high temperature result in a small variation in bending angle under load, but in a greater difference in final bending angle after unloading, so important difference in springback at room and high temperature is observed. In Fig. 14, springback (\%) after unload calculated at room temperature and at maximum temperature distribution (up to $770^{\circ} \mathrm{C}$ ), versus punch displacement, is presented. This calculation corresponds with sheet thickness $6 \mathrm{~mm}$ and die aperture $48 \mathrm{~mm}$. Springback curve at high 


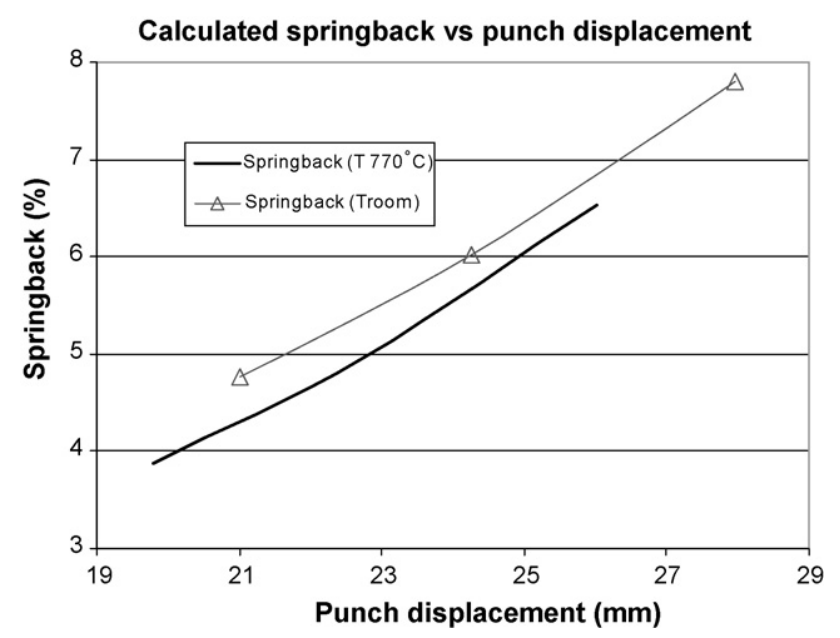

Fig. 14 - Calculated springback (\%) vs. punch displacement at room temperature and maximum temperature $770^{\circ} \mathrm{C}$ (die aperture $48 \mathrm{~mm}$ ).

temperature is clearly under the curve at room temperature. With the same punch displacement, final angle is smaller in the heated sheet, so as temperature increases, springback is reduced. A test was carried out in described conditions (sheet thickness $6 \mathrm{~mm}$, temperature distribution up to $770^{\circ} \mathrm{C}$ ). Punch displacement was $22.6 \mathrm{~mm}$ and springback (\%) was $6 \%$. From Fig. 12, corresponding springback at this displacement was $4.9 \%$, and so, difference between calculated and measured is $1.1 \%$.

Error in springback angle after unload was calculated comparing measured angles and values predicted by analytical model in the rest of tests, corresponding to other sheet thickness and other temperature distributions.

As a resume error was inferior to $1.5 \%$.

\section{Conclusions}

In this paper experimental validation of an analytical thermomechanical model for air bending at high temperature is presented. Non-conventional experimental set up was projected and fabricated in order to carry out bending tests at room temperature and with a temperature distribution up to $770^{\circ} \mathrm{C}$. Experimental work demonstrated that air bending at high temperature is possible and relatively easy to implement in industry.

Temperature measurements in thermal tests showed that thermal model is able to predict with very good accuracy (errors less than 2\%) temperature distribution, once heating parameters are fixed.

Bending tests allowed to measure bending force versus punch displacement and springback angle, at room and high temperature. Calculated errors (less than $5 \%$ in predicted bending force and less than $1.5 \%$ in predicted springback angle) showed good accuracy of the bending model both at room and elevated temperature. As it is expected, high temperature along bend length results in reduced bending force and springback.
The work presented in this paper could help to improve bending processes in industry. Firstly, flexibility in processes is important because of small batch sizes and short lead times in sheet metal forming, and it could be achieved using accurate models to predict process parameters as an inexpensive and fast tool. On the other hand forming at elevated temperature, could increase ranges of use of machines and tools. As an example, thicker sheets can be bent in a machine when they are previously heated, because of decrease in maximum bending force. Also, metal sheet manufacture at high temperature could improve processes when improved mechanical properties of material workpiece are involved, as in the case of high strength steels, recently implemented in automobile industry.

However, the relatively complex mathematical formulation of the models, do not facilitate the widespread use of analytical models in industry. A software easy to use in production departments, should be developed as a complementary work of an analytical model.

\section{REF E R E N C E S}

Asnafi, N., 2000. Springback and fracture in v-die air bending of thick stainless steel sheets. Mater. Des. 21, 217-236.

Bilal, M.A., McCuen, R.H., 1996. Numerical Methods for Engineers. Prentice-Hall International, Londres.

J.A. Canteli, Air bending process of stainless steel with local heating, PhD Thesis, University Carlos III of Madrid, 2003.

Canteli, J.A., Cantero, J.L., Miguélez, M.H., 2006. Development of software for easy prediction of process parameters in air bending with local heating. Int. J. Vehicle Des. 42, 170-197.

de Vin, L.J., 2000. Curvature prediction in air bending of metal sheet. J. Mater. Process. Technol. 100, 257-261.

de Vin, L.J., 2001. Expecting the unexpected, a must for accurate brake forming. J. Mater. Process. Technol. 117, 244-248.

de Vin, L.J., Streppel, A.H., Singh, U.P., Kals, H.J.J., 1996. A process model for air bending. J. Mater. Process. Technol. 57, 48-54.

Eary, D.F., Reed, E.A., 1974. Techniques of Pressworking Sheet Metal-An Engineering Approach to Die Design, 2nd ed. Prentice-Hall, Englewood Cliffs, NJ, pp. 67-72.

Forcellese, A., Fratini, L., Gabrielli, F., Micari, F., 1998. The evaluation of springback in 3D stamping and coining processes. J. Mater. Process. Technol. 80-81, 108-112.

Hennige, T., 2000. Development of irradiation strategies for 3D-laser forming. J. Mater. Process. Technol. 103, 102-108.

Hu, Z., Labudovic, M., Wang, H., Kovacevic, R., 2001. Computer simulation and experimental investigation of sheet metal bending using laser beam scanning. Int. J. Mach. Tools Manuf. 41, 589-607.

Huang, Y.M., Leu, D.K., 1998. Effects of process variables on V-die bending process of steel sheet. Int. J. Mech. Sci. 40 (7), 631-650.

Hultquist, P.F., 1988. Numerical Methods for Engineers and Computer Scientists. Benjamin-Cummings, Menlo Park.

Inamdar, M.V., Date, P.P., Sabnis, S.V., 2002. On the effects of geometric parameters on springback in sheets of five materials subjected to air vee bending. J. Mater. Process. Technol. 123, 459-463.

E.E. Jones, Apparatus and method of compensating for springback in a workpiece, United States Patent 4802357 (1989).

Khan, A.S., Huang, S., 1995. Continuum Theory of Plasticity. John Wiley \& Sons, New York. 
Livatyali, H., Altan, T., 2001. Prediction and elimination of springback in strait flanging using computer aided design methods. Part 1. Experimental investigations. J. Mater. Process. Technol. 117, 262-268.

Melin, L., Akerberg, L., Ohman, U., 1982. Sheet Metal Forming, University Textbook in Sheet Metal Forming, 5th ed. Lulea University of Technology, p. 128.
Moon, Y.H., Kang, S.S., Cho, J.R., Kim, T.G., 2003. Effect of tool temperature on the reduction of the springback of aluminium sheets. J. Mater. Process. Technol. 132, 365-368.

$\mathrm{Z}$. Tan, Analysis and modelling of plastic bending processes, $\mathrm{PhD}$ Thesis, Technological University of Lulea, Sweden, 1994.

Timoshenko, 2002. Resistencia de Materiales. Thomson, Madrid. 